\title{
Modelo para treinamento simulado de anastomoses cirúrgicas por laparoscopia
}

\section{Model for simulated training of surgical anastomoses by laparoscopy}

Márcio Alencar Barreiraํ․ Delano Gurgel Silveiraํ. Annya Costa Araújo de Macedo Goes².

1 Mestre em Tecnologia Minimamente Invasiva e Simulação na Área da Saúde, Centro Universitário Christus (Unichristus). Cirurgião Geral, Hospital Universitário Walter Cantídio (HUWC), Maternidade Escola Assis Chateaubrian (MEAC), Empresa Brasileira de Serviços Hospitalares (EBSERH), Fortaleza, Ceará, Brasil. 2 Doutorado em Cirurgia, Professora do Departamento de Cirurgia Geral, Universidade Federal do Ceará (UFC), Fortaleza, Ceará, Brasil.

\section{RESUMO}

Objetivo: apresentar um modelo, de baixo custo e prático, para treinamento por simulação de uma anastomose cirúrgica por laparoscopia. Metodologia: Estudo exploratório, com a utilização de pesquisa bibliográfica. Resultados: O modelo é prático e barato. Conclusão: O modelo apresentado pode ser utilizado no ensino da cirurgia laparoscópica.

Palavras-chave: Anastomose cirúrgica. Treinamento por simulação. Laparoscopia.

\section{ABSTRACT}

Objective: to present a low cost and practical model for training by simulation of a surgical anastomosis by laparoscopy. Methods: Exploratory study, with the use of bibliographic research. Results: The model is practical and cheap. Conclusion: The model presented can be used in the teaching of laparoscopic surgery.

Keywords: Surgical anastomosis. Training by simulation. Laparoscopy.

Autor correspondente: Márcio Alencar Barreira, Avenida Beira Mar, 2780, Meireles, Fortaleza, Ceará. CEP: 60165-121. Telefone: +55 85 99151-4975. E-mail: marciofmj@msn.com

Conflito de interesses: Não há qualquer conflito de interesses por parte de qualquer um dos autores.

Recebido em: 22 Nov 2017; Revisado em: 17 Mai 2017; Aceito em: 17 Mai 2017. 


\section{INTRODUÇÃO}

Durante o longo aprendizado da cirurgia laparoscópica, o número e a severidade de complicações intraoperatórias geralmente são maiores do que as observadas em cirurgias abertas. ${ }^{1}$ Como forma de reduzir estas complicações, os programas de simulação permitem um ambiente seguro e eficiente para adquirir habilidades cirúrgicas desejadas. ${ }^{2}$

No Brasil, os cerca de 200 programas de residência médica na área de cirurgia e suas mais de 1000 vagas ofertadas, não são capazes de oferecer, na sua grande maioria, educação cirúrgica suficiente na área da cirurgia minimamente invasiva para seus residentes. ${ }^{3}$ Uma pesquisa com cinquenta residentes de áreas cirúrgicas do Hospital São Paulo mostrou que somente 20\% estavam satisfeitos com o volume cirúrgico. ${ }^{4}$

A introdução de um modelo de treinamento para residentes com interesse em laparoscopia pode ser viável para países em desenvolvimento, dando a oportunidade de hospitais com recursos limitados fornecerem um meio de treinamento cirúrgico que possa influir positivamente na curva de aprendizado dos residentes de cirurgia geral ${ }^{5}$ e, assim, reduzir custos hospitalares. ${ }^{6}$

Um simulador do tipo caixa preta (CP) é uma opção com menor custo e, portanto, acessível à maioria dos residentes em todo o mundo. ${ }^{5,7} \mathrm{O}$ preço é um fator muito importante a ser analisado, já que muitas vezes tecnologias médicas não podem ser oferecidas em locais onde os recursos financeiros são limitados. O simulador de realidade virtual é uma opção de alto custo com manutenção dispendiosa e de limitada disponibilidade no Brasil. ${ }^{7}$

Visando aumentar a confiança e melhorar a formação dos residentes de Cirurgia Geral na confecção de anastomoses laparoscópicas, criou-se um modelo de treinamento de baixo custo e prático que pode ser utilizado no interior de uma caixa preta.

\section{METODOLOGIA}

Foi realizado um estudo exploratório, com a utilização de pesquisa bibliográfica. A pesquisa exploratória é muito utilizada para realizar um estudo preliminar sobre determinado assunto e, assim, familiarizar-se com o fenômeno que está sendo investigado, de modo que a pesquisa subsequente possa ser concebida com uma maior compreensão e precisão.

A pesquisa bibliográfica foi realizada através da pesquisa de artigos pelo Portal da Capes utilizando as palavras anastomose cirúrgica, treinamento por simulação e laparoscopia. Os artigos que não tinham relação com o ensino de procedimentos cirúrgicos por simulação não foram utilizados.

Buscou-se propor um modelo de treinamento a ser utilizado por residentes de Cirurgia Geral do Hospital Universitário Walter Cantídio durante as aulas práticas de simulação laparoscópica, que ocorrem em um laboratório de treinamento simulado localizado no bloco da pós-graduação.

\section{RESULTADOS}

Trata-se de um modelo que pode simular anastomoses digestivas através do uso de materiais com custo aproximado de US\$ 1 por anastomose confeccionada, já que são utilizados 2 balões simples de aniversário fixados a uma placa com 2 tachinhas em cada balão a 2 centímetros de distância do orifício a ser anastomosado (Figura 1).

O fio a ser utilizado fica a critério do treinamento proposto, sendo o fio de seda 3.0 adequado e com baixo custo. A forma de realizar a anastomose pode ser feita com uma sutura contínua (Figura 2) ou com pontos simples, mas é importante ter sempre um auxiliar para ajudar na execução das tarefas.

Figura 1. Modelo de treinamento de anastomoses digestivas por laparoscopia.

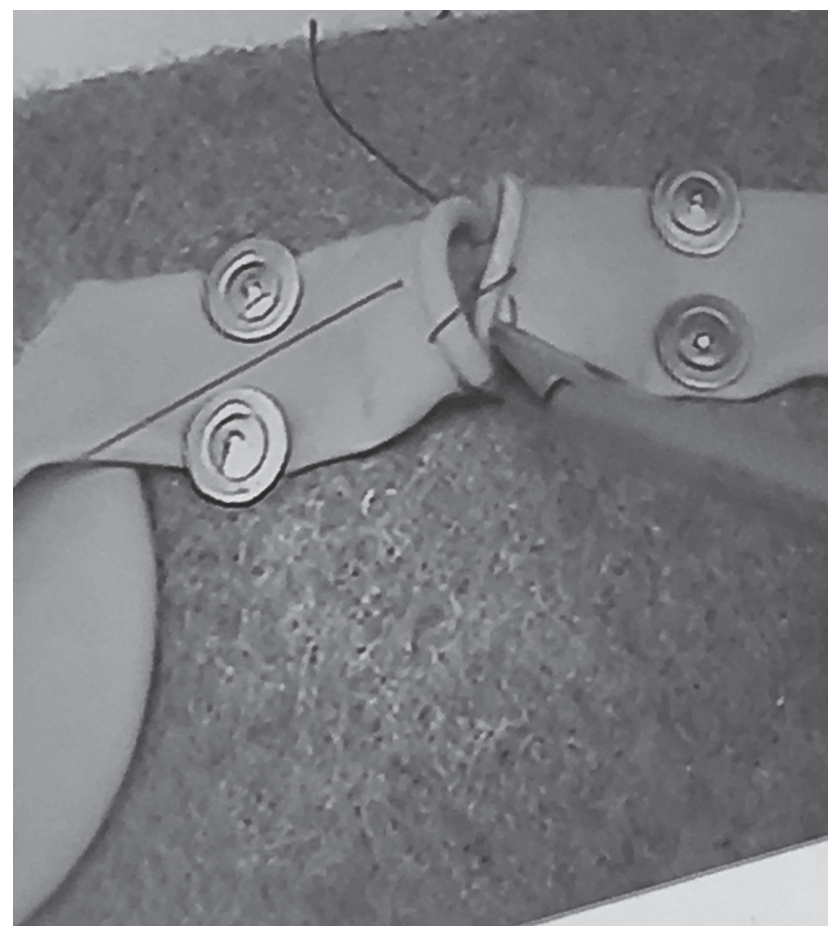

Figura 2. Anastomose realizada utilizando uma sutura contínua.

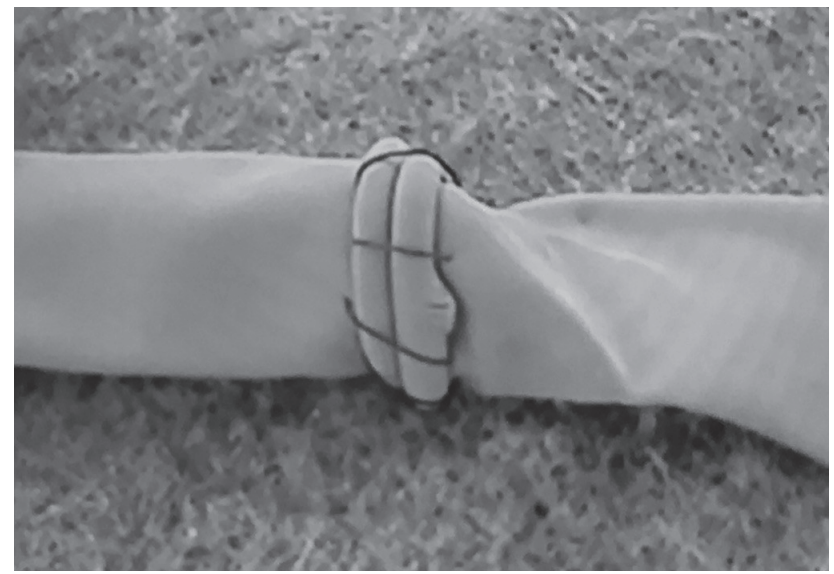




\section{DISCUSSÃO}

A preocupação com a formação cirúrgica após a residência vem causando discussões sobre o currículo de treinamento cirúrgico. ${ }^{8,9}$ Um estudo realizado com 186 cirurgiões mostrou que existe a necessidade de se criar um currículo para o treinamento simulado de habilidades laparoscópicas avançadas, já que a maioria dos entrevistados não tem proficiência em anastomoses intestinais. Os entrevistados acharam que o currículo deveria depender da viabilidade e da capacidade dos médicos em repetirem os exercícios quantas vezes fossem necessárias. ${ }^{10}$

O programa de treinamento em cirurgia minimamente invasiva proporciona um elevado grau de satisfação aos seus participantes, e os capacita a realizar procedimentos técnicos mais complexos, como as suturas, além de melhorar seu desempenho profissional. ${ }^{11}$ Um treinamento de anastomoses em órgãos sintéticos realizado com doze residentes de cirurgia geral mostrou que após a confecção de dez anastomoses, divididas em cinco sessões em um período de seis semanas,

\section{REFERÊNCIAS}

1. Kuhry E, Van Veen RN, Langeveld HR, Steyerberg EW, Jeekel J, Bonjer HJ. Open or endoscopic total extraperitoneal inguinal hernia repair? A systematic review. Surg Endosc. 2007;21(2):161-6.

2. Aggarwal R, Darzi A. Simulation to enhance patient safety: why aren't we there yet? Chest. 2011;140(4):854-8.

3. Ferreira EA, Rasslan S. Surgical education in Brazil. World J Surg. 2010;34(5):880-3.

4. Herbella FA, Fuziy RA, Takassi GF, Dubecz A, Del Grande JC. Avaliação do treinamento e expectativas profissionais em residentes de cirurgia. Rev Col Bras Cir. 2011;38(4):280-4.

5. Long KL, Spears C, Kenady DE, Roth JS. Implementation of a low-cost laparoscopic skills curriculumina third world setting. J Surg Educ. 2014;71(6):860-4.

6. Bashankaev B, Baido S, Wexner SD. Review of available methods of simulation training to facilitate surgical education. Surg Endosc. 2011;25(1):28-35.

7. Gravante G, Venditti D. Systematic review on low-cost box models to achieve basic and advanced laparoscopic skills during modern surgical training. Surg Laparosc Endosc Percutan Tech. 2013;23(2):109-20. foi possível adquirir habilidades básicas e avançadas em cirurgia laparoscópica. ${ }^{12}$

Segundo Cristancho, Moussa e Dubrowski, ${ }^{13}$ a simulação cirúrgica necessita identificar um objetivo, sistematizar um treinamento levando em consideração os recursos disponíveis, utilizar ferramentas de avaliação de desempenho e realizar avaliações com intuito de validar a eficácia do programa educacional proposto.

\section{CONCLUSÃO}

A criação de um modelo de baixo custo de produção para o treinamento de anastomoses laparoscópicas mostra-se como uma opção viável para o treinamento de residentes em instituições médicas com poucos recursos financeiros. No entanto, o currículo de treinamento ideal para o treinamento utilizando balões ainda deve ser estabelecido em pesquisas futuras levando em consideração o método utilizado para a confecção das anastomoses.

8. Mattar SG, Alseidi AA, Jones DB, Jeyarajah DR, Swanstrom LL, Aye RW, et al. General surgery residency inadequately prepares trainees for fellowship: results of a survey of fellowship program directors. Ann Surg. 2013;258(3):440-9.

9. Nakayama DK, Taylor SM. SESC Practice committee survey: surgical practice in the duty-hour restriction era. Am Surg. 2013;79(7):711-5.

10. Nepomnayshy D, Alseidi AA, Fitzgibbons SC, Stefanidis D. Identifying the need for and content of an advanced laparoscopic skills curriculum: results of a national survey. Am J Surg. 2016;211(2):4215.

11. Loureiro MP, Claus CM, Bonin EA, Cury A Filho, Dimbarre $\mathrm{D}$, Trauczinski $\mathrm{P}$, et al. Long-term professional performance of minimally invasive surgery post-graduates. Rev Col Bras Cir. 2015;42(2):130-5.

12. Barreira MA, Siveira DG, Rocha HA, Moura LG Junior, Mesquita CJ, Borges GC. Model for simulated training of laparoscopic gastroenterostomy. Acta Cir Bras. 2017;32(1):81-9.

13. Cristancho SM, Moussa F, Dubrowski A. A framework-based approach to designing simulation augmented surgical education and training programs. Am J Surg. 2011;202(3):344-51.

\section{Como citar:}

Barreira MA, Silveira DG, Goes AC. Modelo para treinamento simulado de anastomoses cirúrgicas por laparoscopia. Rev Med UFC. 2018 jul-set;58(3):65-67. 